\title{
Resveratrol analogue 3,4,4'-trihydroxy-trans-stilbene induces apoptosis and autophagy in human non- small-cell lung cancer cells in vitro
}

\author{
Lu ZHANG ${ }^{1, *}$, Fang DAI ${ }^{2}$, Pan-long SHENG ${ }^{1}$, Zhi-qiang $\mathrm{CHEN}^{1}$, Qi-ping XU ${ }^{1}$, Yu-qi GUO ${ }^{3, *}$ \\ ${ }^{1}$ College of Bioengineering, He-nan University of Technology, Lianhua Street, Zhengzhou 450001, China; ${ }^{2}$ State Key Laboratory \\ of Applied Organic Chemistry, Lanzhou University, Lanzhou 730000, China; ${ }^{3}$ Department of Obstetrics and Gynecology, The Third \\ Affiliated Hospital of Zhengzhou University, Zhengzhou 450052, China
}

\begin{abstract}
Aim: To investigate the effects of 3,4,4'-trihydroxy-trans-stilbene (3,4,4'-THS), an analogue of resveratrol, on human non-small-cell lung cancer (NSCLC) cells in vitro.

Methods: Cell viability of NSCLC A549 cells was determined by MTT assay. Cell apoptosis was evaluated using flow cytometry and TUNEL assay. Cell necrosis was evaluated with LDH assay. The expression of apoptosis- or autophagy-associated proteins was measured using Western blotting. The formation of acidic compartments was detected using AO staining, neutral red staining and Lysotracker-Red staining. LC3 punctae were analyzed with fluorescence microscopy.

Results: Treatment with 3,4,4'-THS (10-80 $\mu \mathrm{mol} / \mathrm{L})$ concentration-dependently inhibited the cell viability. It did not cause cell necrosis, but induced apoptosis accompanied by up-regulation of cleavaged PARP, caspase3/9 and Bax, and by down-regulation of Bcl-2 and surviving. It also increased the formation of acidic compartments, LC3-II accumulation and GFP-LC3 labeled autophagosomes in the cells. It inhibited the mTOR-dependent pathway, but did not impair autophagic flux. 3,4,4'-THS-induced cell death was enhanced by the autophagy inhibitors 3-MA ( $5 \mathrm{mmol} / \mathrm{L})$ or Wortmannin $(2 \mu \mathrm{mol} / \mathrm{L})$. Moreover, 3,4,4'-THS treatment elevated the ROS levels in the cells, and co-treatment with 3-MA further elevated the ROS levels. 3,4,4'-THS-induced apoptosis and autophagy in the cells was attenuated by NAC (10 $\mathrm{mmol} / \mathrm{L})$

Conclusion: 3,4,4'-THS induces both apoptosis and autophagy in NSCLC A549 cells in vitro. Autophagy inhibitors promote 3,4,4'-THSinduced apoptosis of A549 cells, thus combination of 3,4,4'-THS and autophagy inhibitor provides a promising strategy for NSCLC treatment.
\end{abstract}

Keywords: 3,4,4-trihydroxy-trans-stilbene; resveratrol; non-small-cell lung cancer; apoptosis; autophagy; mTOR; 3-MA; Wortmannin; NAC

Acta Pharmacologica Sinica (2015) 36: 1256-1265; doi: 10.1038/aps.2015.46; published online 20 Jul 2015

\section{Introduction}

Lung cancer is a leading cause of cancer death, accounting for approximately $26 \%$ of all female and $28 \%$ of all male cancer deaths in $2013^{[1]}$. Non-small-cell lung cancer (NSCLC), including squamous carcinoma, adenocarcinoma, and large cell carcinoma, accounts for more than $80 \%$ of all lung cancers and is associated with poor prognosis and poor median survival ${ }^{[2]}$. Over half of these patients are not eligible for surgery at the time of diagnosis, and chemotherapy remains one of the most common therapies, even though it is associated with a range of side effects ${ }^{[3]}$. The discovery and development of more potent compounds with fewer side effects for the treatment of

\footnotetext{
* To whom correspondence should be addressed.

E-mail chaperones@163.com (Lu ZHANG); yuqi-guo@163.com (Yu-qi GU0)

Received 2015-01-08 Accepted 2015-04-23
}

NSCLC has become an increasingly active area of research.

Natural products (NPs) are believed to be an important source of new chemical substances that can serve as potent therapeutics for various human cancers, including NSCLC ${ }^{[4]}$. The use of NPs as anti-tumor agents for the management of NSCLC is an attractive idea because they are readily available and exhibit little or no toxicity ${ }^{[4]}$. Resveratrol (3,5,4-trihydroxy-trans-stilbene, RSV), a phytoalexin found in the skin of grapes, peanuts, mulberries, red wine, Polygonum cuspidatum Sieb et Zucc and many types of foods, is one such NP and has been shown to exhibit both anti-tumor and chemopreventive effects $^{[5]}$. Previous studies have indicated that resveratrol functions not only as a chemoprotective agent in human NSCLC A549 cells but also has therapeutic effects against existing lung carcinoma $^{[6,7]}$. Although high doses of resveratrol can inhibit the proliferation of A549 cells by inducing apoptosis and 
autophagic cell death ${ }^{[8,9]}$, a major challenge for the use of this compound is that the concentration of resveratrol required to induce cancer cell death in vitro is too high to achieve in vivo in a clinical setting ${ }^{[10-12]}$.

The structural modification of NPs is an efficient method to increase their activity and reduce the side effects. A number of synthetic analogues of resveratrol have been developed in recent years, and some hydroxystilbenes with improved activity have been discovered. For example, 3,4,5-trihydroxytrans-stilbene $(3,4,5-\mathrm{THS})$ has been shown to exhibit a potent cytotoxic effect on human leukemia Jurkat cells by inducing extensive cell apoptosis at lower concentrations than resveratrol $^{[13]}$. The anti-proliferative effects and cytotoxicity of 4,4'-dihydroxy-trans-stilbene $\left(4,4^{\prime}\right.$-DHS), which were attributed to the inhibition of DNA polymerase delta activity and DNA replication, have been shown to be more efficient than those of resveratrol ${ }^{[14,15]}$. Changing the 5-hydroxy group in resveratrol to 4-hydroxy (3,4,4'-trihydroxy-trans-stilbene, 3,4,4'-THS; Figure 1) dramatically increases the protective effects of the resveratrol analogues against free radical-induced lipid peroxidation, photo-sensitized DNA oxidative damage, and the free radical-induced hemolysis of human red blood cells ${ }^{[16]}$. Furthermore, 3,4,4' -THS reportedly is a more efficient antiproliferative agent than resveratrol in human leukemia HL-60 cells ${ }^{[16]}$. However, the mechanism by which $3,4,4^{\prime}$-THS kills cancer cells remains unclear. Our preliminary studies demonstrated that 3,4,4'-THS induced extensive cell death and cytoplasmic vacuolation in A549 cells. These findings prompted our interest in investigating the effects of 3,4,4'-THS on A549 cells, as well as the mechanisms that facilitates this effect.

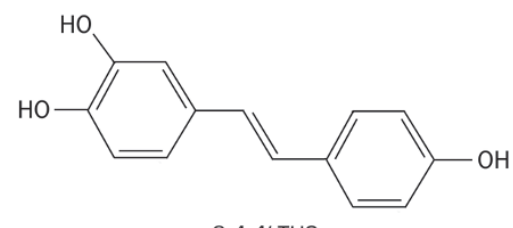

$3,4,4^{\prime}-\mathrm{THS}$

Figure 1. The chemical structure of 3,4,4'-THS. The molecular formula of this compound is $\mathrm{C}_{14} \mathrm{H}_{12} \mathrm{O}_{3}$, and its molecular weight is $228.2433 \mathrm{~g} / \mathrm{mol}$.

\section{Materials and methods}

\section{Cell culture and 3,4,4'-THS treatment}

$3,4,4^{\prime}$-THS (purity was at least $99 \%$ by HPLC analysis) was provided by Prof Bo ZHOU (State Key Laboratory of Applied Organic Chemistry, Lanzhou University). 3,4,4'-THS was dissolved in DMSO $(0.1 \mathrm{~mol} / \mathrm{L})$ to generate a stock solution. A549 cells were cultured in RPMI-1640 medium (Gibco, Carlsbad, CA, USA, 11875-093) containing 10\% heat-inactivated fetal bovine serum (FBS, HyClone, Logan, UT, USA, SV30087.02), penicillin (100 U/mL) and streptomycin (100 $\mathrm{mg} / \mathrm{mL}$ ) in a humidified incubator at $37^{\circ} \mathrm{C}$ in $5 \% \mathrm{CO}_{2}$. The cells were treated with concentrations of 3,4,4'-THS ranging from 10 to $80 \mu \mathrm{mol} / \mathrm{L}$ supplemented with 5\% FBS for 3, 6, or $12 \mathrm{~h}$.

\section{Cell viability assay}

A549 cells were plated in 96-well cell culture plates. 3-Methyladenine (3-MA, Sigma-Aldrich, M9281) and N-acetylcystein (NAC, Sigma-Aldrich, St Louis, MO, USA, A7250) were dissolved in sterile water. Wortmannin (Sigma-Aldrich, St Louis, MO, USA, W1628) was dissolved in DMSO. When cells reached $80 \%$ confluency, they were washed once with basal RPMI-1640 medium (Gibco, Carlsbad, CA, USA, 31800022). The cells were then treated with various compounds, as indicated in the legends to the figures. The cell viability was determined with an MTT assay as described previously ${ }^{[17]}$. The viability (\%) was expressed as $=($ optical density $[O D]$ of treated group/OD of control group) $\times 100 \%$. The viability of the control group was set to $100 \%$.

\section{Lactate dehydrogenase (LDH) assay}

The cell culture medium was collected after the A549 cells were treated with DMSO or 3,4,4'-THS (10-80 $\mu \mathrm{mol} / \mathrm{L})$ for $12 \mathrm{~h}$. An LDH assay was performed using an LDH kit (Nanjing Jiancheng Co, Nanjing, China) according to the manufacturer's protocol.

\section{Flow cytometry for Annexin V/propidium iodide (PI) double staining}

A549 cells were treated with various concentrations of 3,4,4'THS (10-80 $\mu \mathrm{mol} / \mathrm{L})$ for $12 \mathrm{~h}$ and then washed with PBS. The number of apoptotic cells was measured using the annexin V-FITC/PI apoptosis detection kit (DOJINDO Biotechnology, Shanghai, China, AD10) according to the manufacturer's protocol. The data were acquired and analyzed using flow cytometry (BD FACSCalibur) and the Cell Quest software.

\section{Western blot analysis}

After treatment, the cells were lysed in lysis buffer containing 25 mmol/L Tris-HCl (pH 6.8), 2\% SDS, 6\% glycerol, 1\% 2-mercaptoethanol, $2 \mathrm{mmol} / \mathrm{L}$ PMSF, 0.02\% bromophenol blue and a protease inhibitor cocktail (Sigma-Aldrich, St Louis, MO, USA, P8340) for $10 \mathrm{~min}$ at room temperature and boiled for an additional $10 \mathrm{~min}$. The total endothelial protein extracts (30 $\mu \mathrm{g}$ ) were separated by $12 \%$ or $15 \%$ SDS-polyacrylamide gel electrophoresis and transferred to a polyvinylidene difluoride (PVDF) membrane (Millipore, Billerica, MA,USA, ISEQ09120). The membrane was blocked with $5 \%(w / v)$ nonfat dry milk in PBS-Tween 20 (PBST; 0.05\%) for $1 \mathrm{~h}$ and then incubated with the primary antibodies (1:10000 in PBST) at $4{ }^{\circ} \mathrm{C}$ overnight. After 3 washes in PBST, the PVDF membrane was incubated with the appropriate HRP-conjugated secondary antibodies (1:20000) for $1 \mathrm{~h}$ at room temperature. The immunoreactive bands were developed with the Pierce ECL (Thermo Fisher Scientific, Waltham, MA, USA, 32106) Western blotting system. The relative quantity of the proteins was analyzed with the Quantity One software (Bio-Rad, Hercules, CA, USA). The primary antibodies against PARP (sc-56197), Bcl-2 (sc492), Bax (sc-20067), and Survivin (sc-10811) were purchased from Santa Cruz Biotechnology(Dallas, TX, USA). The primary antibodies against LC3B (L7543) and $\beta$-actin (A1978) 
were purchased from Sigma-Aldrich(St Louis, MO, USA). The primary antibody against p62 (610833) was purchased from BD Transduction Laboratories(San Diego, CA, USA). The primary antibodies against p-p70S6K (9206), p70S6K (9202), p-4EBP1 (9456), 4EBP1 (9452), cleaved caspase-3 (9661), and cleaved caspase-9 (9509) were purchased from Cell Signaling Technology(Beverly, MA, USA). The secondary HRP-conjugated anti-rabbit IgG (sc-2004) and anti-mouse IgG (sc-2371) antibodies were purchased from Santa Cruz Biotechnology(Dallas, TX, USA).

\section{Acridine orange $(\mathrm{AO})$ staining}

AO moves freely across biological membranes and produces red fluorescence in acidic compartments and green fluorescence in the cytosolic and nuclear compartments. After treatment, cells were stained with AO $(5 \mu \mathrm{g} / \mathrm{mL}$, Sigma, St Louis, $\mathrm{MO}, \mathrm{USA}, \mathrm{A} 6014)$ at $37^{\circ} \mathrm{C}$ for $1 \mathrm{~min}$ and observed by fluorescence microscopy (Nikon, Tokyo, Japan).

\section{Neutral red uptake assay}

After treatment, cells were stained with neutral red $(0.05 \%$ in phenol red-free RPMI-1640/BSA 0.1\%), a membrane-permeable dye that accumulates in acidic compartments, at $37^{\circ} \mathrm{C}$ for $5 \mathrm{~min}$. The cells were then washed and observed with an inverted phase contrast microscope (Nikon, Tokyo, Japan).

\section{Double-staining with Lysotracker-Red and Hoechst 33258}

After treatment, cells were double-stained with $1 \mathrm{mmol} / \mathrm{L}$ Lysotracker-Red DND (Invitrogen, L7528) and $10 \mu \mathrm{mol} / \mathrm{L}$ Hoechst 33258 (Sigma, 14530) at $37^{\circ} \mathrm{C}$ for $20 \mathrm{~min}$. The cells were then washed and observed by fluorescence microscopy (Nikon, Tokyo, Japan). Apoptotic cells were stained bright blue due to chromatin condensation. The percentage of fluorescently stained cells was calculated based on the images.

\section{LC3B punctae analysis}

A549 cells were plated in 48-well culture dishes and transduced with Premo ${ }^{\mathrm{TM}}$ Autophagy Sensor LC3B-GFP (Invitrogen, Carlsbad, CA, USA, P36235) for $24 \mathrm{~h}$. The cells were then treated with concentrations of 3,4,4'-THS (10-80 $\mu \mathrm{mol} / \mathrm{L})$ for 3 , 6, or $12 \mathrm{~h}$, respectively. A549 cells were observed by fluorescence microscopy (Nikon, Japan).

Terminal deoxynucleotidyl transferase-mediated dUTP nick endlabeling (TUNEL) assay

After the A549 cells were treated with various compounds as indicated in the legends to the figures, the number of apoptotic cells was measured with the TUNEL (Promega, Madison, WI, USA, G3250) assay according to the manufacturer's protocol. Cells were evaluated with a fluorescence microscope. The apoptotic rate was quantified according to the TUNELpositive rate.

\section{Measurement of reactive oxygen species (ROS)}

DCFH-DA, a non-fluorescent probe, can permeate cell membranes and is cleaved by esterase to yield non-fluorescent DCFH. DCFH is oxidized in the presence of ROS to generate highly fluorescent DCF. After treatment with various compounds as indicated in the legends to the figures, A549 cells were incubated with $10 \mu \mathrm{mol} / \mathrm{L}$ DCFH-DA (Sigma, St Louis, $\mathrm{MO}$, USA, D6883) for $30 \mathrm{~min}$ at $37^{\circ} \mathrm{C}$, and the fluorescent intensity of DCF was measured using a Epoch ${ }^{\mathrm{TM}}$ spectrophotometer. A cell-free condition was used to determine the background fluorescence, and the relative fluorescence intensity in the control group was set to 1 .

\section{Statistical analyses}

All experiments were performed in duplicate and repeated at least three times. The data are expressed as the mean \pm standard error of the mean (SEM). The differences between groups were analyzed with a one-way analysis of variance (ANOVA), and the means of two groups were compared using Student's $t$-test with SPSS version 17.0 (SPSS Inc, Chicago, IL, USA). Differences were considered statistically significant at $P<0.05$.

\section{Results}

THS potently induced apoptosis in A549 cells

To investigate the cytotoxic effect of $3,4,4^{\prime}$-THS (Figure 1) on A549 cells, phase-contrast microscopy was used to evaluate the changes in the morphology of A549 cells treated with different concentrations of 3,4,4'-THS (10-80 $\mu \mathrm{mol} / \mathrm{L})$ for various times $(3,6$, or $12 \mathrm{~h})$. As shown in Figure 2A, 3,4,4'-THS increased the number of shrinking cells that cells detached from the bottom of culture dish. The MTT assay showed that 3,4,4'-THS inhibited cell viability in a dose- and time-dependent manner (Figure 2B). These data indicate that 3,4, $4^{\prime}$-THS induced A549 cell death. Notably, the number and size of large vacuoles in A549 cells increased in response to 10-40 $\mu \mathrm{mol} / \mathrm{L} \mathrm{3,4,4^{ \prime } - \mathrm { THS }}$ prior to the 6-h time point.

To determine the mechanism of 3,4,4'-THS-induced cell death, we detected whether 3,4,4'-THS induced necrosis or apoptosis in A549 cells. The results of the LDH assay showed that the LDH activity did not significantly differ between the control group and those treated with 3,4,4'-THS for $12 \mathrm{~h}$ (Figure 2C). However, the treatment of A549 cells with 3,4,4'THS for $12 \mathrm{~h}$ significantly increased the percentage of annexin V-FITC-positive cells (Figure 2D). These results suggested that $3,4,4^{\prime}$-THS induced apoptosis, but not necrosis, in A549 cells.

To further demonstrate that cell death was due to apoptosis, the effects of 3,4,4'-THS on apoptosis-associated proteins were detected. The Western blot analyses showed that the cleavages of PARP, caspase 3/9 and Bax increased in 3,4,4'-THStreated cells compared with control cells, whereas the expression of anti-apoptotic proteins, such as Bcl-2 and survivin, decreased (Figure 2E and 2F). These data indicated that 3,4,4'THS triggered cell death in A549 cells by inducing apoptosis, which was associated with the up-regulation of pro-apoptotic 
A
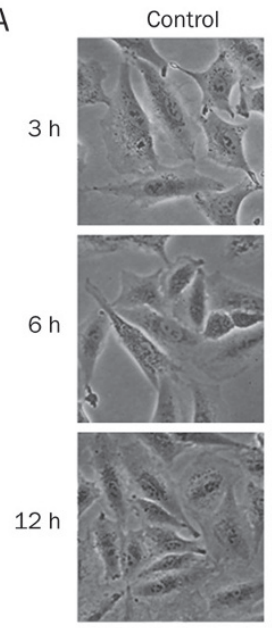

10
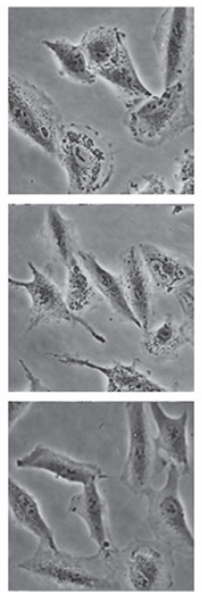

20
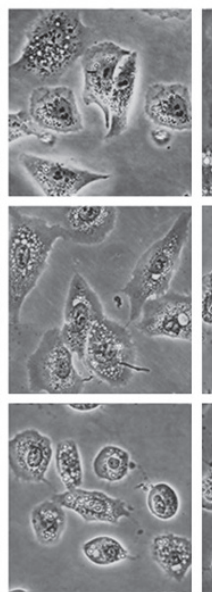

40
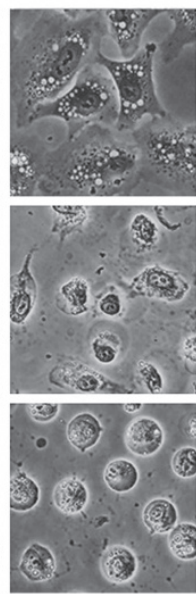

$80(\mu \mathrm{mol} / \mathrm{L})$
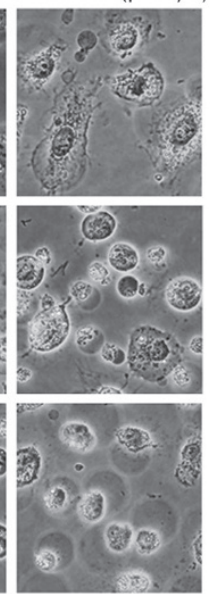

B

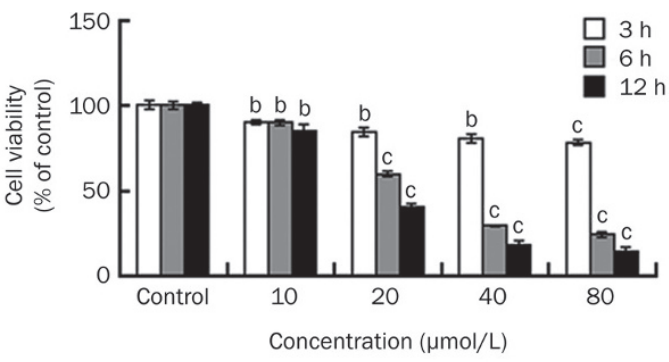

D

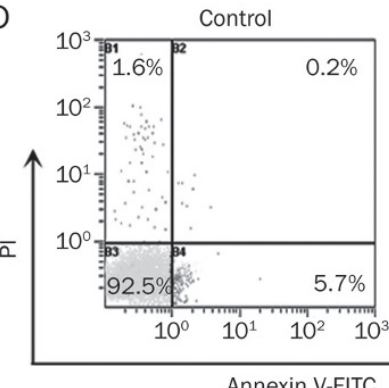

E

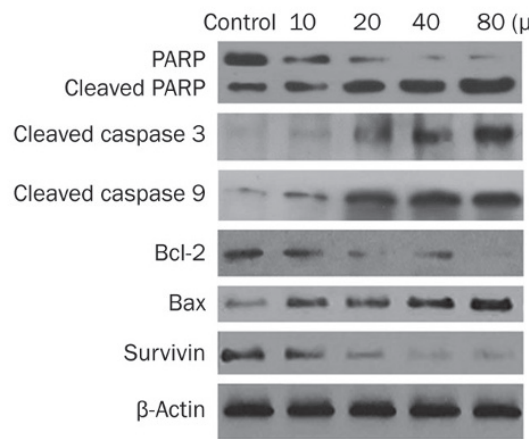

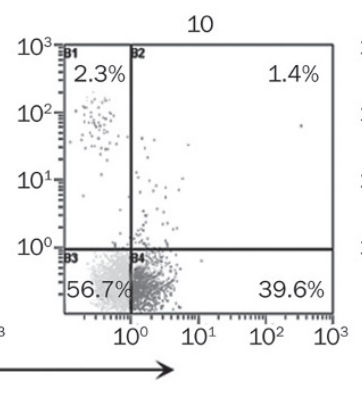
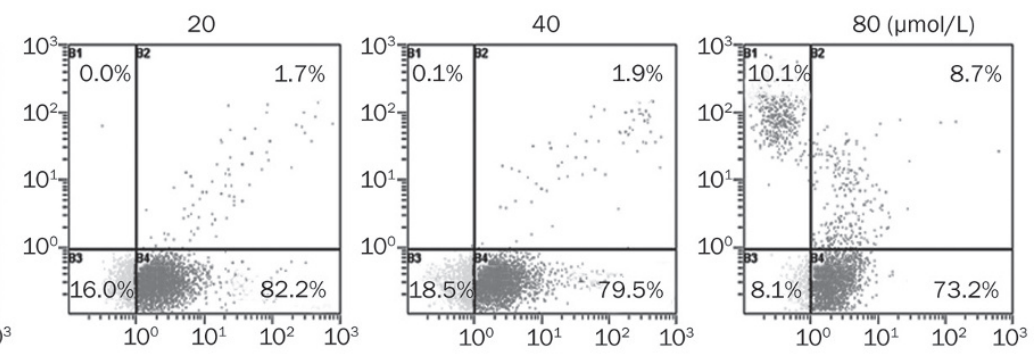

F
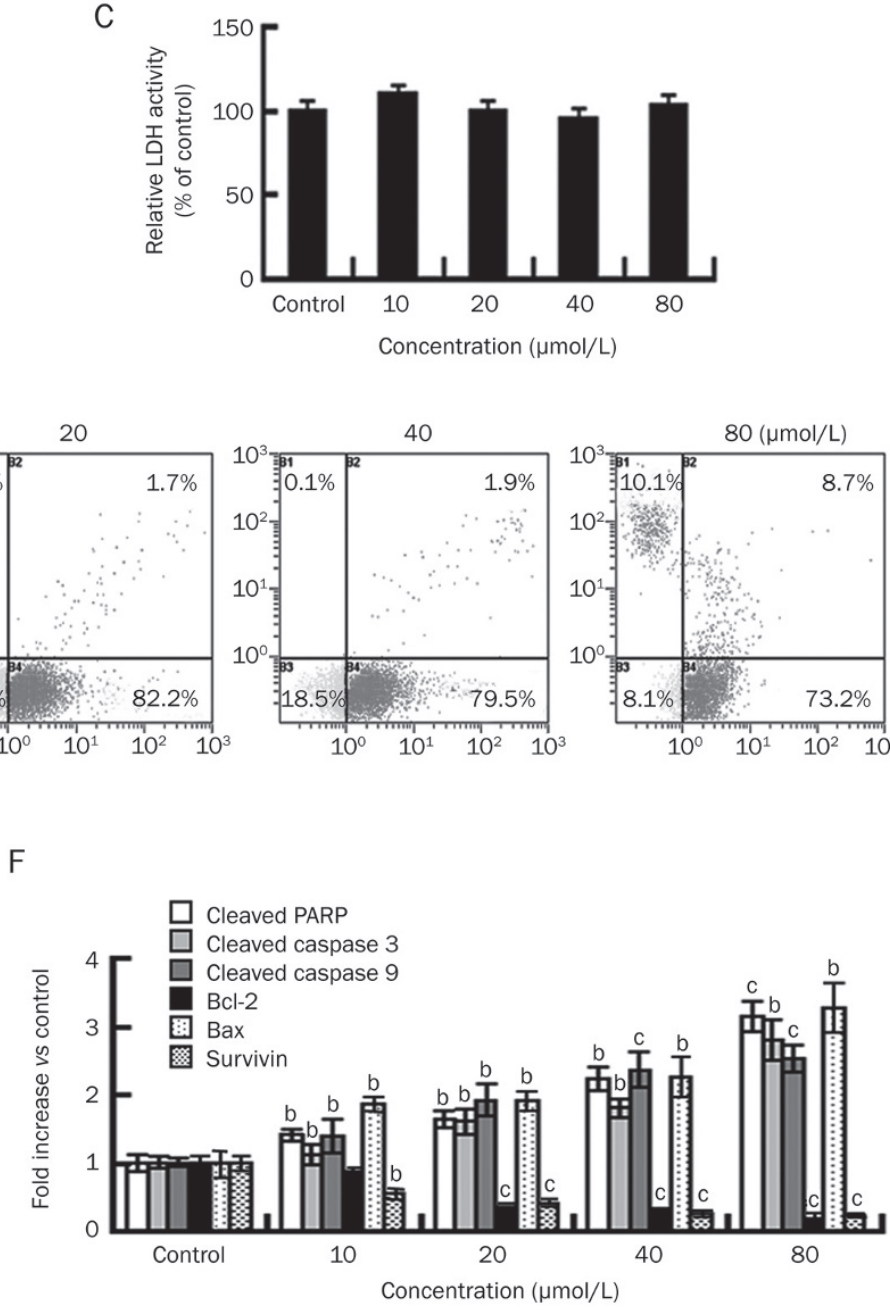

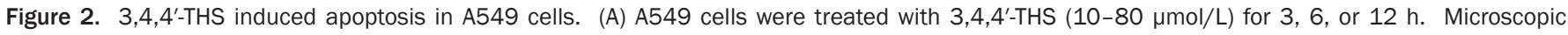
photographs (400x) were taken under an inverted phase contrast microscope. (B) Cell viability determined by MTT assay. (C) The relative LDH activity

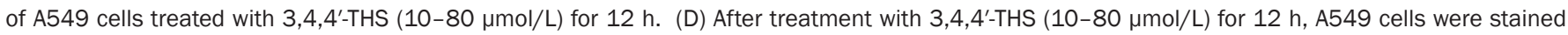
with Annexin V-FITC/PI and then analyzed by flow cytometry. Annexin V-positive cells were considered to be in the early stage of apoptosis, whereas Annexin $\mathrm{V}$ and Pl-positive cells were considered to be in the late stage of apoptosis. (E) Western blot analysis of apoptosis-provoking proteins in A549 cells treated with 3,4,4'-THS (10-80 $\mu \mathrm{mol} / \mathrm{L})$ for $12 \mathrm{~h}$. (F) Histogram shows the relative level of proteins. Data are expressed as the mean ( \pm SEM) of three independent experiments. ${ }^{b} P<0.05,{ }^{c} P<0.01$ vs control. 
proteins and down-regulation of anti-apoptotic proteins.

\section{3,4,4'-THS increased the formation of acidic compartments}

To understand the nature of the vacuoles that were observed under phase-contrast microscopy, we first stained cells with AO. A549 cells treated with 10-40 $\mathrm{mmol} / \mathrm{L} \mathrm{3,4,4}$ '-THS for $3 \mathrm{~h}$ displayed many fluorescent red dots in the cytoplasm, which represented acidic compartments (such as lysosomes and autophagolysosome). The highest concentration of 3,4,4' ${ }^{\prime}$ THS (80 $\mathrm{\mu mol} / \mathrm{L})$ induced strong morphological changes associated with apoptosis, including chromatin condensation and nuclear fragmentation (Figure 3A); therefore, fluorescent red dots could not be detected. Nuclear condensation and fragmentation were evident for longer treatment periods (6 or $12 \mathrm{~h}$ ), even at a dose of $40 \mu \mathrm{mol} / \mathrm{L}$. Similar results were obtained from the neutral red staining (Figure 3B). To confirm the effects of $3,4,4^{\prime}$-THS on the induction of acidic compartments in A549 cells, double staining with Lysotracker-Red (an organelleselective, fluorescent probe that labels and tracks acidic organelles) and Hoechst 33258 was performed. 3,4,4'-THS treatment increased the intensity of LysoTracker-red-stained vesicles in A549 cells compared with control cells (Figure 3C). Increasing the concentration or treatment time resulted in nuclear cleavage and chromatin condensation (Figure 3C and 3D), which confirmed that $3,4,4^{\prime}$-THS triggered apoptosis in A549 cells.

\section{3,4,4'-THS induced autophagy in A549 cells}

The aforementioned findings prompted us to address the possibility that the acidic compartments were indeed autophagolysosomes and that 3,4,4'-THS induced an autophagic as well as an apoptotic response in A549 cells. We first investigated the effect of 3,4,4'-THS on LC3 processing and LC3II accumulation with a Western blot analysis. LC3 processing, the ratio of LC3-II/ $\beta$-actin, was markedly enhanced in A549 cells treated with 3,4,4'-THS (10-40 $\mu \mathrm{mol} / \mathrm{L})$ for 3 or $6 \mathrm{~h}$ (Figure 4A and 4B). Additionally, 10-40 $\mu \mathrm{mol} / \mathrm{L} \mathrm{3,4,4^{ \prime } - \mathrm { THS }}$ increased the number of GFP-LC3-labeled autophagosomes in A549 cells (Figure 4C and 4D). At $80 \mu \mathrm{mol} / \mathrm{L}, 3,4,4^{\prime}$-THS strongly induced A549 cell apoptosis, and enhanced LC3 accumulation and GFP-LC3-labeled autophagosomes could conse-
A
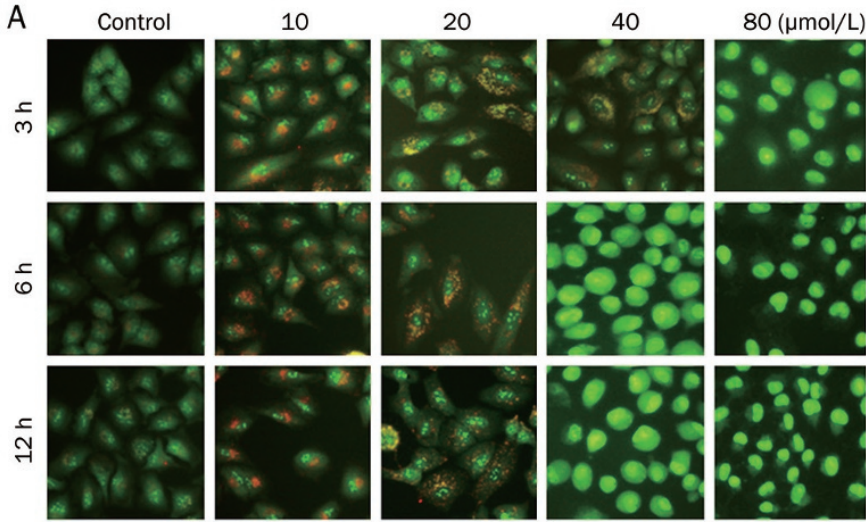

B

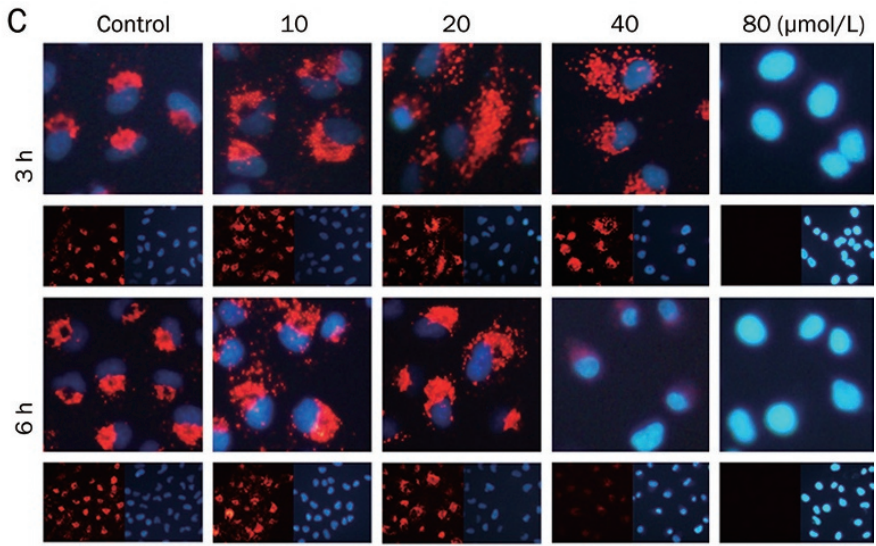

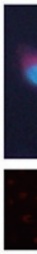


D

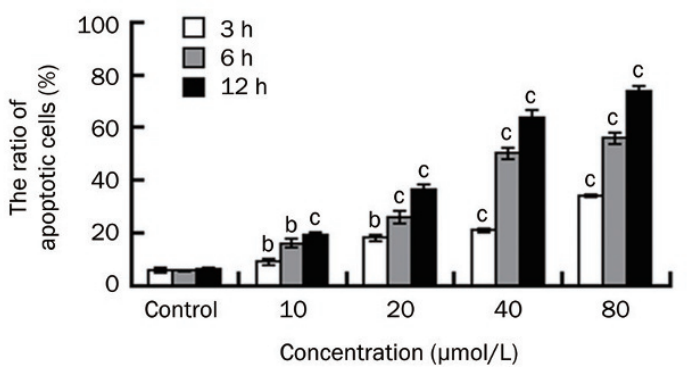

Figure 3. 3,4,4'-THS increased the acidic compartments in A549 cells. (A) Immunofluorescent photographs (100x) show the AO staining of A549 cells treated with 3,4,4'-THS (10-80 $\mu \mathrm{mol} / \mathrm{L})$ for 3,6 , or $12 \mathrm{~h}$. (B) Microscopic photographs (200x) show the neutral red staining of A549 cells treated with 3,4,4'-THS (10-80 $\mathrm{mol} / \mathrm{L})$ for 3, 6, or $12 \mathrm{~h}$. (C) Immunofluorescent photographs (400x) show the Lysotracker-red and Hoechst 33258 staining of A549 cells treated with 3,4,4'-THS (10-80 $\mu \mathrm{mol} / \mathrm{L})$ for 3, 6, or $12 \mathrm{~h}$. (D) Histogram shows the ratio of apoptotic A549 cells. Data are expressed as the mean $\left( \pm\right.$ SEM) of three independent experiments. ${ }^{b} P<0.05,{ }^{\circ} P<0.01$ vs control. 
A

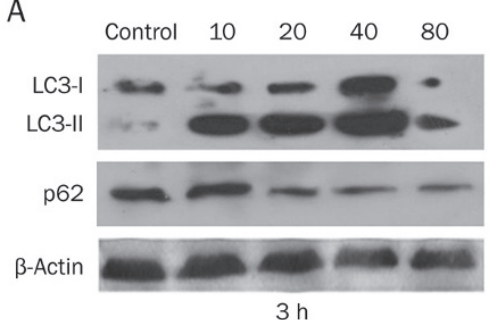

C
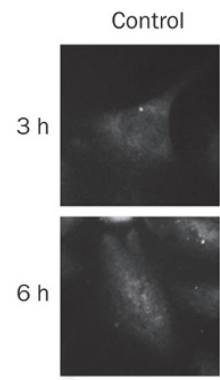

10


Control $10 \quad 20 \quad 40 \quad 80(\mu \mathrm{mol} / \mathrm{L})$

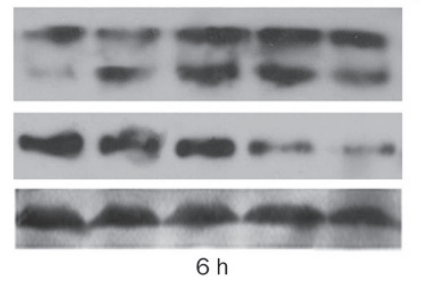

$6 \mathrm{~h}$
B

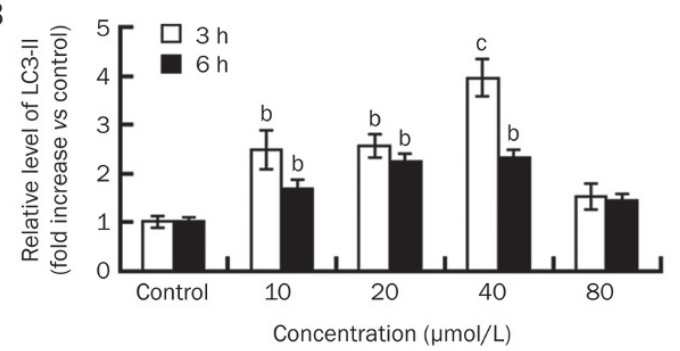

E

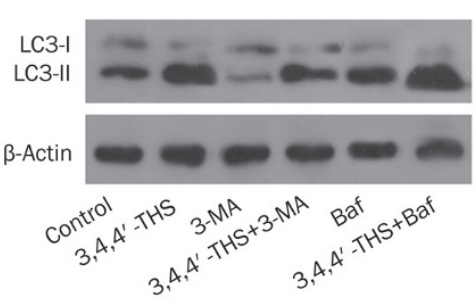

20
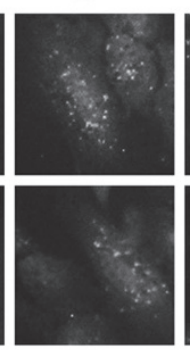

40

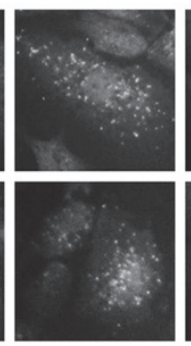

$80(\mu \mathrm{mol} / \mathrm{L})$

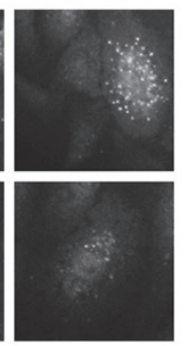

D



G

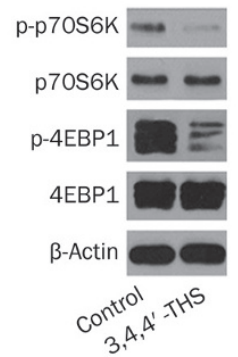

$\mathrm{H}$



Figure 4. 3,4,4'-THS induced autophagy in A549 cells. (A) Western blot analysis of LC3-II and p62 in A549 cells treated with 3,4,4'-THS (10-80 $\mu$ mol/ L) for 3 or 6 h. (B) Histogram shows the relative level of LC3-II. (C) Immunofluorescent photographs (200x) shows the endogenous punctuate of LC3 in A549 cells. Nuclei were stained with Hoechst 33258. (D) Histogram shows the quantification of endogenous LC3 punctuate per cell. (E) Western blot analysis of LC3-II in A549 cells treated with 3,4,4'-THS ( $40 \mu \mathrm{mol} / \mathrm{L})$ for $3 \mathrm{~h}$. 3-MA ( $5 \mathrm{mmol} / \mathrm{L})$ or bafilomycin A1 (100 $\mathrm{nmol} / \mathrm{L})$ was added to the cells for $1 \mathrm{~h}$ prior to 3,4,4'-THS treatment. (F) Histogram show the relative level of LC3-II. (G) Western blot analysis of p-p70S6K and p-4EBP1 in A549 cells treated without or with 3,4,4'-THS $(40 \mu \mathrm{mol} / \mathrm{L})$ for $3 \mathrm{~h}$. (H) Histogram shows the relative level of $\mathrm{p}-\mathrm{p} 70 \mathrm{~S} 6 \mathrm{~K}$ and $\mathrm{p}-4 \mathrm{EBP} 1$. Data are expressed as the mean ( \pm SEM) of three independent experiments. ${ }^{b} P<0.05,{ }^{c} P<0.01$ vs control. ${ }^{\mathrm{e}} P<0.05$ vs $3,4,4^{\prime}-\mathrm{THS},{ }^{\mathrm{h}} P<0.05$ vs Baf.

quently not be detected.

The increases in the LC3-II levels and autophagosome accumulation induced by 3,4,4'-THS may be related to either enhanced autophagosome formation due to enhanced autophagic activity or reduced autophagosome degradation due to impaired autophagic flux ${ }^{[18]}$. To discriminate between these two possibilities, we first evaluated the level of p62, which is involved in the formation of autophagosomes and constitutively degraded by the autophagic pathway via specific binding to $\mathrm{LC}^{[19]}$. Therefore, a decrease in the p62 level reflects unimpaired autophagic flux. Our results showed that 3,4,4'THS decreased the level of p62 (Figure 4A), indicating that this compound does not impair autophagic flux. Furthermore, the class III PtdIns3K inhibitor 3-MA, an early-stage autophagy inhibitor, suppressed the accumulation of LC3-II induced by
3,4, $4^{\prime}$-THS (Figure 4E and 4F). If 3,4,4' ${ }^{\prime}$ THS induces autophagy, co-treatment with late-stage autophagy inhibitor should further increase the level of LC3-II. Thus, we utilized bafilomycin A1 (Baf), an inhibitor of the vacuolar $\mathrm{H}^{+}$-ATPase, to inhibit autophagic flux. Our results showed that co-treatment with 3,4,4'-THS (40 $\mathrm{mmol} / \mathrm{L})$ and Baf enhanced the accumulation of LC3-II compared with cells treated with 3,4,4'-THS alone (Figure 4E and 4F).

Both mTOR-dependent and -independent pathways can induce autophagy in cancer cells ${ }^{[20,21]}$. To determine whether mTOR signaling is associated with 3,4,4'-THS-induced autophagy, we examined the phosphorylation status of p70S6K and 4EBP1, two targets of mTOR, after 3,4,4'-THS treatment. As shown in Figure $4 \mathrm{G}$ and $4 \mathrm{H}, 3,4,4^{\prime}$-THS (40 $\left.\mu \mathrm{mol} / \mathrm{L}\right)$ attenuated the phosphorylation of p70S6K and 4EBP1. 
A



B

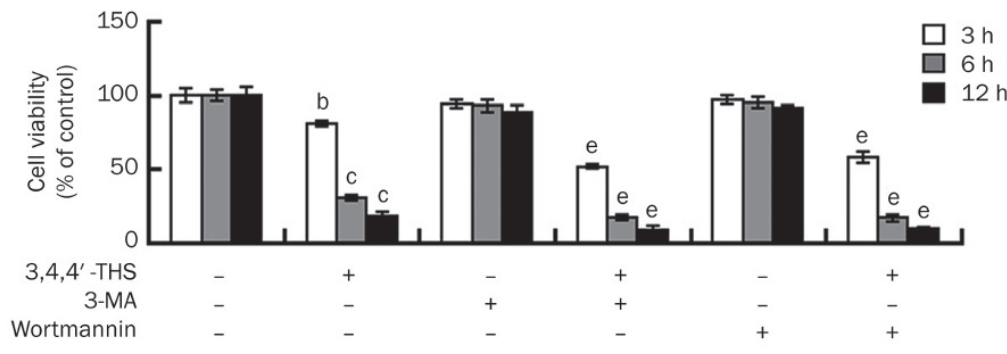

C

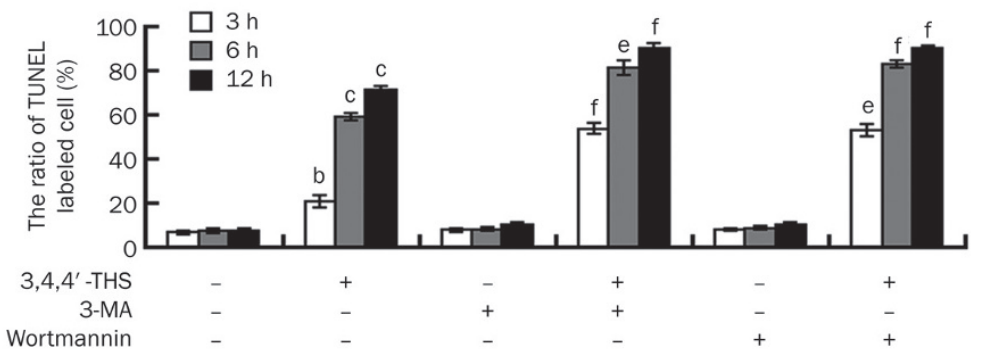

D

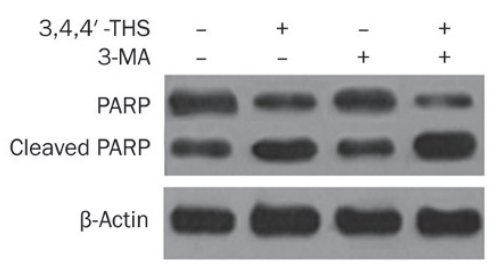

E

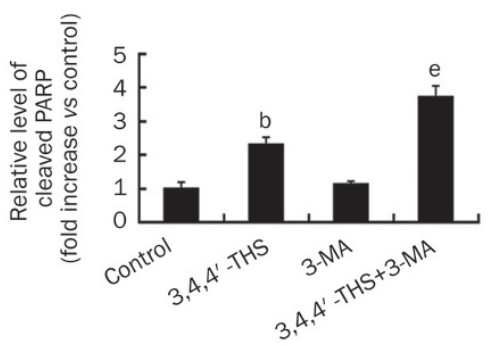

F

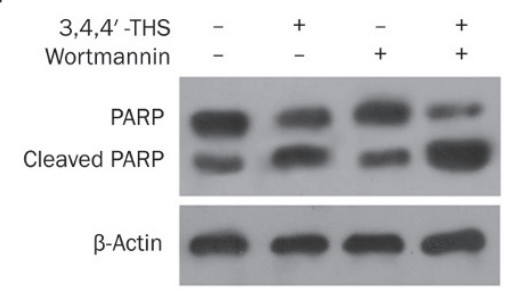

G

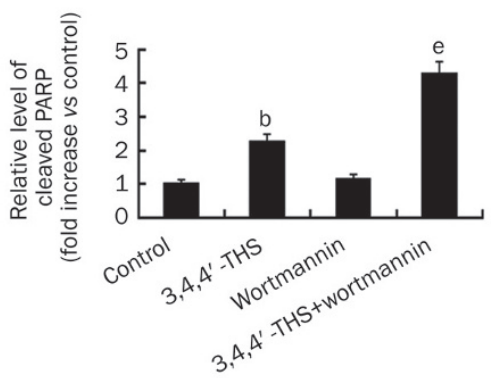

Figure 5. Inhibition of autophagy enhanced the toxicity of 3,4,4'-THS in A549 cells. (A) A549 cells were treated with 3,4,4'-THS (40 $\mu$ mol/L), 3-MA (5 $\mathrm{mmol} / \mathrm{L})$, wortmannin $(2 \mu \mathrm{mol} / \mathrm{L})$ or a combined treatment of 3,4,4'-THS and 3-MA or wortmannin for 3, 6, or $12 \mathrm{~h}$. Microscopic photographs (400x) were taken under an inverted phase contrast microscope. (B) Cell viability determined by MTT assay. (C) Histogram shows the ratio of TUNEL-positive cells. (D) Western blot analysis of cleaved PARP in A549 cells treated without or with 3,4,4'-THS (40 $\mu$ mol/L) for $3 \mathrm{~h}$. 3-MA was added to the cells for $1 \mathrm{~h}$ prior to 3,4,4'-THS treatment. (E) Histogram shows the relative level of cleaved PARP. (F) Western blot analysis of cleaved PARP in A549 cells treated without or with 3,4,4'-THS $(40 \mu \mathrm{mol} / \mathrm{L})$ for $3 \mathrm{~h}$. Wortmannin was added to the cells for $1 \mathrm{~h}$ prior to 3,4,4'-THS treatment. (G) Histogram shows the relative level of cleaved PARP. Data are expressed as the mean ( \pm SEM) of three independent experiments. ${ }^{\mathrm{b}} P<0.05,{ }^{\mathrm{c}} P<0.01$ vs control. ${ }^{\mathrm{e}} P<0.05$, ${ }^{\mathrm{f}} P<0.01$ vs $3,4,4^{\prime}$-THS.

Inhibition of autophagy promoted 3,4,4'-THS-induced apoptosis in A549 cells

Because autophagy can result in both cell survival and death, we further investigated the mechanism of the cytotoxic effect of 3,4,4'-THS-induced autophagy on A549 cells. Phasecontrast microscopy was used to evaluate changes in the morphology of A549 cells co-treated with 3,4,4'-THS (40 $\mu \mathrm{mol} / \mathrm{L})$ and autophagy inhibitors for various times $(3,6$, or $12 \mathrm{~h})$. Cotreatment with 3,4,4'-THS and 3-MA or wortmannin increased the number of shrinking cells that cells detached from the culture dish compared with A549 cells exposed to only 3,4,4'-THS (Figure 5A). Parallel MTT and TUNEL assays also showed that co-treatment with 3,4,4'-THS and autophagy inhibitors significantly decreased cell viability and induced cell apoptosis compared with the cells treated with only 3,4,4'-THS (Figure 5B and 5C). Moreover, the inhibition of autophagy by 3-MA or wortmannin enhanced the cleavage of PARP-1 (Figure 5D-5G), indicating that 3,4,4'-THS evoked cytoprotective 
A

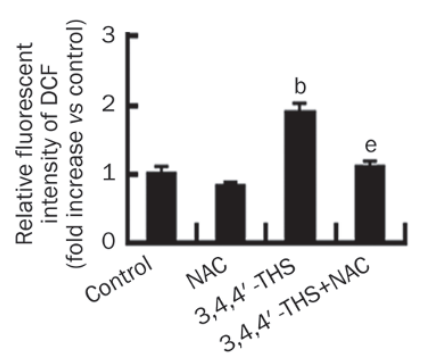

E

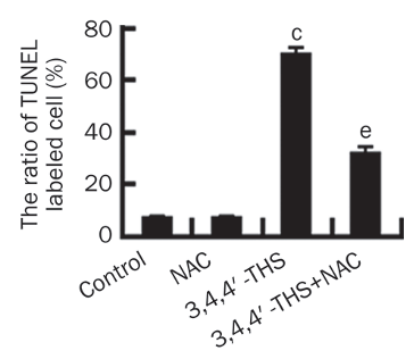

B

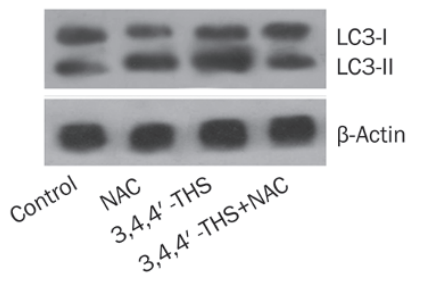

F

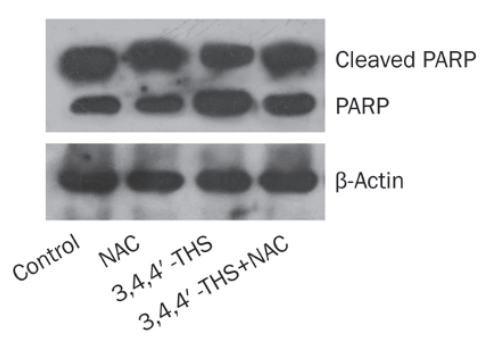

C



G

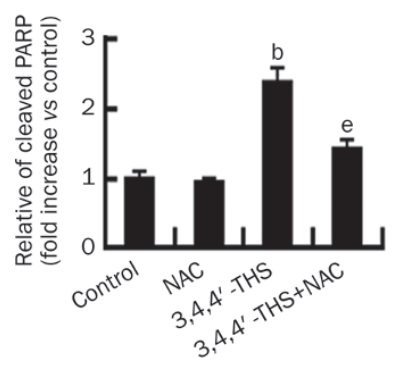

D



$\mathrm{H}$

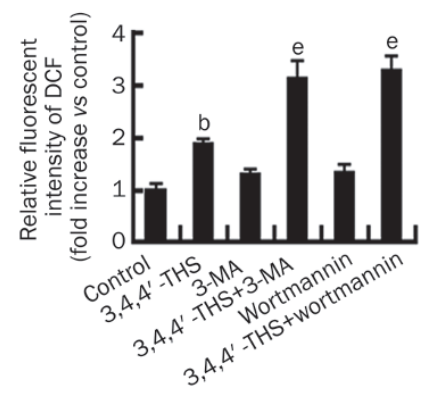

Figure 6. ROS was involved in 3,4,4'-THS induced autophagy and apoptosis in A549 cells. A549 cells were treated with 3,4,4'-THS (40 $\mu$ mol/L), NAC (10 $\mathrm{mmol} / \mathrm{L}$ ) or a combined treatment of 3,4,4'-THS and NAC for $12 \mathrm{~h}$. (A) Relative DCF fluorescence intensity, which corresponds to the ROS production level, measured with a microplate spectrophotometer. (B) Western blot analysis of LC3-II. (C) Histogram shows the relative level of LC3-II. (D) Cell viability determined by the MTT assay. (E) Histogram shows the ratio of TUNEL-positive cells. (F) Western blot analysis of cleaved PARP. (G) Histogram shows the relative level of cleaved PARP. (H) A549 cells were treated with 3,4,4'-THS (40 $\mu \mathrm{mol} / \mathrm{L}), 3-\mathrm{MA}(5 \mathrm{mmol} / \mathrm{L})$, wortmannin $(2 \mu \mathrm{mol} / \mathrm{L})$ or a combined treatment of 3,4,4'-THS and 3-MA or Wortmannin for $12 \mathrm{~h}$. The histogram shows the relative fluorescent intensity of DCF. Data are expressed as the mean $( \pm \mathrm{SEM})$ of three independent experiments. ${ }^{\mathrm{b}} P<0.05,{ }^{\mathrm{c}} P<0.01$ vs control. ${ }^{\mathrm{e}} P<0.05,{ }^{\mathrm{f}} P<0.01$ vs $3,4,4^{\prime}-\mathrm{THS}$.

autophagy, which mitigated apoptosis in A549 cells.

\section{ROS was involved in 3,4,4'-THS induced autophagy and apoptosis} in A549 cells

To verify that ROS was involved in 3,4,4'-THS-induced autophagy and apoptosis, A549 cells were exposed to 3,4,4'THS (40 $\mu \mathrm{mol} / \mathrm{L})$ for $12 \mathrm{~h}$ and then stained with the specific oxidation-sensitive fluorescent dye, DCFH-DA. 3,4,4'-THS treatment significantly increased the relative fluorescent intensity of DCF, corresponding to an enhanced ROS level, whereas NAC, a ROS scavenger, effectively suppressed the high level of ROS (Figure 6A). Scavenging ROS with NAC blocked the high level of LC3-II induced by 3,4,4'-THS (Figure 6B and 6C). In addition, NAC reversed the 3,4,4'-THS-induced reduction in cell viability, high ratio of TUNEL-positive cells and high levels of cleaved PARP (Figure 6D-6G). Collectively, these results demonstrated that ROS was involved in 3,4,4'-THSinduced autophagy and apoptosis. Moreover, we found that the inhibition of autophagy by 3-MA or wortmannin significantly increased the ROS level in 3,4,4' pared with $3,4,4^{\prime}$-THS treatment alone (Figure $6 \mathrm{H}$ ).

\section{Discussion}

A previous study demonstrated that 3,4,4'-THS (Figure 1), an analogue of resveratrol, is more potent than resveratrol in the prevention of the proliferation of human leukemia HL-60 cells $^{[16]}$. However, information on the anti-tumor activity of $3,4,4^{\prime}$-THS is scarce. In this study, we found that 3,4,4'-THS, whose $\mathrm{IC}_{50}$ is approximately $18 \mu \mathrm{mol} / \mathrm{L}$ after $12 \mathrm{~h}$ of incubation, was much more potent than resveratrol in the inhibition of A549 cell viability (Figure 2 and Supplementary Figure 1). Our data also suggested that the cytotoxic effect of 3,4,4'-THS on A549 cells was primarily due to the induction of apoptosis, not necrosis (Figure 2).

One of the most interesting events in the early stage following treatment with 3,4,4'-THS was the cytoplasmic vacuolation. AO staining, neutral red staining, and LysotrackerRed staining identified these vacuoles as acidic compartments (Figure 3). Furthermore, LC3 accumulation and GFP-LC3labeled autophagosomes were enhanced in 3,4,4' -THS-treated cells. Based on these results, we speculated that in addition to the induction of apoptosis, 3,4,4'-THS may trigger autophagy in A549 cells. However, rather than inducing autophagy, an increase in LC3-II accumulation could instead be due to the blocking of autophagosome fusion with lysosomes and degradation of LC3-II by 3,4,4' -THS. To determine the mechanism of 3,4,4'-THS action, we evaluated autophagic flux in A549 cells. Our results showed that p62 did not accumulate in 3,4,4'-THS-treated cells (Figure 4A). Additionally, 3-MA blocked the accumulation of LC3-II induced by 3,4,4'-THS 
(Figure 4E and 4F). Co-treatment with 3,4,4'-THS and bafilomycin A1 enhanced the accumulation of LC3-II compared with cells treated with 3,4,4'-THS alone (Figure 4E and 4F). Collectively, these data indicated that 3,4,4'-THS induced a high level of LC3-II by enhancing autophagic activity and not by blocking autophagy influx in A549 cells.

One of the classical pathways that controls autophagic activity is mTOR signaling ${ }^{[22]}$. mTOR activation enhances protein translation by phosphorylating its targets p70S6K and 4EBP1 and inhibiting the induction of autophagy ${ }^{[23]}$. Our results showed that 3,4,4'-THS suppressed the phosphorylation of p70S6K and 4EBP1, indicating that mTOR signaling may be involved in 3,4,4'-THS-induced autophagy. In addition to acting as a central checkpoint that negatively regulates autophagy, mTOR/p70S6K is an important intracellular signaling pathway that regulates cell survival and apoptosis ${ }^{[24]}$. We speculated that 3,4,4'-THS may cause early autophagy and late apoptosis by inhibiting the mTOR signaling pathway in A549 cells. However, further studies are needed to verify this hypothesis.

Both apoptosis and autophagy are highly conserved processes that maintain organismal and cellular homeostasis, respectively. While apoptosis fulfills this role by removing damaged or unwanted cells, autophagy maintains cellular homeostasis by recycling selective intracellular organelles and proteins, and thus is considered an important protective mechanism for cancer cells in response to chemotherapy ${ }^{[25]}$. However, autophagy may help to induce apoptosis under certain conditions or lead to a non-apoptotic form of programmed cell death (PCD) called autophagic cell death or autophagyassociated cell death (type II PCD) ${ }^{[26]}$. Thus, the function of autophagy as cell death or cell survival machinery in different cases needs to be established. In our study, the inhibition of autophagy by 3-MA or wortmannin significantly reduced the viability of 3,4,4'-treated A549 cells and enhanced 3,4,4'-THSinduced apoptosis (Figure 5), indicating that autophagy triggered by 3,4,4'-THS was a protective response in A549 cells. Resveratrol could reportedly induce both apoptosis and autophagic cell death in A549 cells $^{[9,27]}$. These observations, coupled with the present findings, indicated that 3,4,4'-THS exerts anti-tumor effects via a mechanism different than that of resveratrol.

ROS, which are cytotoxic, often increase in concentration in cancer cells immediately after exposure to chemotherapeutic agents $^{[28]}$. It is also well established that ROS play a central role in the induction of cell autophagy and apoptosis ${ }^{[29]}$. In this study, 3,4,4'-THS elevated the ROS level in A549 cells. When ROS were scavenged by NAC, both 3,4,4'-THS-induced autophagy and apoptosis were inhibited (Figure 6), which demonstrated that the elevated ROS level triggered by $3,4,4^{\prime}$ THS was responsible for the induction of autophagy and apoptosis. More importantly, the inhibition of autophagy markedly enhanced the ROS level in 3,4,4'-THS-treated A549 cells. Autophagy can reportedly remove damaged mitochondria to limit ROS amplification ${ }^{[30]}$. The mitochondria pathway is a key signaling pathway in apoptosis induction (the intrin- sic cell death pathway). Previous studies have shown that ROS acts upstream of mitochondria-initiated apoptosis by promoting Bax translocation to mitochondria, allowing for the activation of caspase-9/3, which results in PARP cleavage ${ }^{[31]}$. Based on the present findings and previous reports, we speculated that 3,4,4'-THS might trigger the ROS-mediated intrinsic cell apoptosis pathway; in addition, autophagy may alleviate 3,4,4'-THS-induced apoptosis in A549 cells by decreasing the excessive ROS levels. However, this hypothesis needs to be addressed in more detail in our future study.

In conclusion, this work is the first report to reveal that 3,4,4'-THS induced excessive cell death in human NSCLC A549 cells by inducing apoptosis. The anti-tumor effect of $3,4,4^{\prime}$-THS was associated with the up-regulation of pro-apoptotic proteins and down-regulation of anti-apoptotic proteins. 3,4,4'-THS could also induce autophagy in A549 cells. mTOR signaling may be involved in this process. Co-treatment with $3,4,4^{\prime}$-THS and autophagy inhibitors enhanced cell death in A549 cells. The high level of ROS induced by 3,4,4'-THS was essential for the induction of autophagy and apoptosis. Autophagy might delay 3,4,4'-THS-induced apoptosis in A549 cells by suppressing the ROS level. In addition, we found that

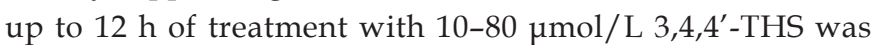
not cytotoxic to normal cultured human umbilical vascular endothelial cells (HUVECs, Supplementary Figure 2). These data suggested that 3,4,4'-THS, together with an autophagy inhibitor might provide a potent strategy for NSCLC treatment. However, several questions remain to be answered. For example, what is the direct target for 3,4,4'-THS on A549 cells? What is the molecular switch between apoptosis and autophagy in response to $3,4,4^{\prime}$-THS? These questions will need to be addressed in more detail in our future studies.

\section{Acknowledgements}

This study was financially supported by the National Natural Science Foundation of China (No 31101001, 31471296, and 21372109), the Program for Science and Technology Innovation Talents in Universities of He-nan Province (No 15HASTIT031), the Key Project of He-nan Educational Committee (No 14A180028), the Foundation for University Key Teacher by He-nan Educational Committee (No 2013GGJS-078) and the Technological innovation incubator program from He-nan University of Technology (№ 11CXRC12).

\section{Author contribution}

$\mathrm{Lu}$ ZHANG and Yu-qi GUO designed the research; Lu ZHANG, Fang DAI, Pan-long SHENG, Zhi-qiang CHEN, and Qi-ping XU performed the research; Lu ZHANG, Fang DAI, Pan-long SHENG, Zhi-qiang CHEN, and Qi-ping XU analyzed the data; Lu ZHANG wrote the paper.

\section{Supplementary information}

Supplementary figures are available at the Acta Pharmacologica Sinica website. 


\section{References}

1 Siegel R, Naishadham D, Jemal A. Cancer statistics 2013. CA Cancer J Clin 2013; 63: 11-30.

2 Ourari-Dhahri B, Ben Slima H, Ben Amar J, El Gharbi L, Ali M, Baccar Azzabi S, et al. Management of non small cell lung cancer. Tunis Med 2012; 90: 847-51.

3 Zhang C, Yang L, Wang XB, Wang JS, Geng YD, Yang CS, et al. Calyxin $Y$ induces hydrogen peroxide-dependent autophagy and apoptosis via JNK activation in human non-small cell lung cancer $\mathrm{NCl}-\mathrm{H} 460$ cells. Cancer Lett 2013; 340: 51-62.

4 Bailly C. Ready for a comeback of natural products in oncology. Biochem Pharmacol 2009; 77: 1447-57.

5 Shukla Y, Singh R. Resveratrol and cellular mechanisms of cancer prevention. Ann N Y Acad Sci 2011; 1215: 1-8.

6 Yin HT, Tian QZ, Guan L, Zhou Y, Huang XE, Zhang H. In vitro and in vivo evaluation of the antitumor efficiency of resveratrol against lung cancer. Asian Pac J Cancer Prev 2013; 14: 1703-6.

7 Zhang W, Wang X, Chen T. Resveratrol induces apoptosis via a Bakmediated intrinsic pathway in human lung adenocarcinoma cells. Cell Signal 2012; 24: 1037-46.

8 Kim YA, Lee WH, Choi TH, Rhee SH, Park KY, Choi YH. Involvement of p21 WAF1/CIP1, pRB, Bax and NF-kappaB in induction of growth arrest and apoptosis by resveratrol in human lung carcinoma A549 cells. Int J Oncol 2003; 23: 1143-9.

9 Zhang J, Chiu J, Zhang H, Qi T, Tang Q, Ma K, et al. Autophagic cell death induced by resveratrol depends on the $\mathrm{Ca}(2+) / \mathrm{AMPK} / \mathrm{mTOR}$ pathway in A549 cells. Biochem Pharmacol 2013; 86: 317-28.

10 Cottart CH, Nivet-Antoine V, Laguillier-Morizot C, Beaudeux JL. Resveratrol bioavailability and toxicity in humans. Mol Nutr Food Res 2010; 54: 7-16.

11 Patel KR, Scott E, Brown VA, Gescher AJ, Steward WP, Brown K. Clinical trials of resveratrol. Ann N Y Acad Sci 2011; 1215: 161-9.

12 Scott E, Steward WP, Gescher AJ, Brown K. Resveratrol in human cancer chemoprevention-choosing the right dose. Mol Nutr Food Res 2012; 56: 7-13.

13 Wang Y, Wang B, Cheng J, Yang L, Liu ZL, Balan K, et al. FADDdependent apoptosis induction in Jurkat leukemia T-cells by the resveratrol analogue, 3,4,5-trihydroxy-trans-stilbene. Biochem Pharmacol 2005; 69: 249-54.

14 Fan GJ, Liu XD, Qian YP, Shang YJ, Li XZ, Dai F, et al. 4,4'-Dihydroxytrans-stilbene, a resveratrol analogue, exhibited enhanced antioxidant activity and cytotoxicity. Bioorg Med Chem 2009; 17: 2360-5.

15 Savio M, Coppa T, Bianchi L, Vannini V, Maga G, Forti L, et al. The resveratrol analogue 4,4'-dihydroxy-trans-stilbene inhibits cell proliferation with higher efficiency but different mechanism from resveratrol. Int J Biochem Cell Biol 2009; 41: 2493-502.

16 Cai W, Zhang L, Song Y, Zhang B, Cui X, Hu G, et al. 3,4,4'-Trihydroxy- trans-stilbene, an analogue of resveratrol, is a potent antioxidant and cytotoxic agent. Free Radic Res 2011; 45: 1379-87.

17 Price $\mathrm{P}$, McMillan TJ. Use of the tetrazolium assay in measuring the response of human tumor cells to ionizing radiation. Cancer Res 1990; 50: 1392-6.

18 Ragazzoni Y, Desideri M, Gabellini C, De Luca T, Carradori S, Secci D, et al. The thiazole derivative CPTH6 impairs autophagy. Cell Death Dis 2013; 4: e524.

19 Bjrky G, Lamark T, Brech A, Outzen H, Perander M, Overvatn A, et al. p62/SQSTM1 forms protein aggregates degraded by autophagy and has a protective effect on Huntingtin-induced cell death. J Cell Biol 2005; 171: 603-14.

20 Li T, Su L, Zhong N, Hao X, Zhong D, Singhal S, et al. Salinomycin induces cell death with autophagy through activation of endoplasmic reticulum stress in human cancer cells. Autophagy 2013; 9: 105768.

21 Tovilovic G, Ristic B, Siljic M, Nikolic V, Kravic-Stevovic T, Dulovic M, et al. mTOR-independent autophagy counteracts apoptosis in herpes simplex virus type 1-infected U251 glioma cells. Microbes Infect 2013; 15: 615-24.

22 Jung $\mathrm{CH}$, Ro SH, Cao J, Otto NM, Kim DH. mTOR regulation of autophagy. FEBS Lett 2010; 584: 1287-95

23 Wang L, Dong Z, Huang B, Zhao B, Wang H, Zhao J, et al. Distinct patterns of autophagy evoked by two benzoxazine derivatives in vascular endothelial cells. Autophagy 2010; 6: 1115-24.

24 Owonikoko TK, Khuri FR. Targeting the PI3K/AKT/mTOR pathway: biomarkers of success and tribulation. Am Soc Clin Oncol Educ Book 2013. doi: 10.1200/EdBook_AM.2013.33.e395.

25 YJ Fan, WX Zong, The cellular decision between apoptosis and autophagy. Chin J Cancer 2013; 32: 121-9.

26 Hasima N, Ozpolat B. Regulation of autophagy by polyphenolic compounds as a potential therapeutic strategy for cancer. Cell Death Dis 2014; 5: e1509.

27 Weng CJ, Yang YT, Ho CT, Yen GC. Mechanisms of apoptotic effects induced by resveratrol, dibenzoylmethane, and their analogues on human lung carcinoma cells. J Agric Food Chem 2009; 57: 5235-43.

28 Schumacker PT. Reactive oxygen species in cancer cells: live by the sword, die by the sword. Cancer Cell 2006; 10: 175-6.

29 Kaminskyy VO, Zhivotovsky B. Free radicals in cross talk between autophagy and apoptosis. Antioxid Redox Signal 2014; 21: 86-102.

30 Azad MB, Chen Y, Gibson SB. Regulation of autophagy by reactive oxygen species (ROS): implications for cancer progression and treatment. Antioxid Redox Signal 2009; 11: 777-90.

31 Wang GW, Lv C, Shi ZR, Zeng RT, Dong XY, Zhang WD, et al. Abieslactone induces cell cycle arrest and apoptosis in human hepatocellular carcinomas through the mitochondrial pathway and the generation of reactive oxygen species. PLoS One 2014; 9: e115151. 